\title{
Degré de Romanité d'un droit successoral coutumier (XIII-XIXe s.) : approche interdisciplinaire d'un corpus de sources numériques
}

\author{
Arnaud Besson, Université de Yale/Adrien Wyssbrod Université de Cambridge
}

Fondation des sources du droit suisse

\section{Introduction}

Depuis 1894, la Collection des sources du droit suisse (SDS) s'est donné comme mission d'éditer les sources juridiques anciennes. En 2014, les SDS ont entrepris de produire des éditions numériques, afin de faciliter l'accès à ces ressources et à leur utilisation pour les chercheurs. L'un des projets pionniers portant sur la coutume de l'ancienne principauté de Neuchâtel est sur le point d'aboutir. Afin de tester le nouveau portail et d'entrevoir les possibilités de recherche qu'il offre, cet article se propose de procéder à une recherche sur la coutume neuchâteloise, en interrogeant directement son degré de romanité par le biais de quelques brèves études de cas.

Actuel canton suisse, Neuchâtel fut de 1504 à 1707 une principauté avec à sa tête la famille française des OrléansLongueville. La maison prussienne des Hohenzollern leur succède jusqu'en 1806, lorsque Frédéric-Guillaume III de Prusse, à la suite de la victoire française à Austerlitz, signe un traité d'échange incluant Neuchâtel et diverses possessions éparses contre le Hanovre. Napoléon ler cède le territoire à l'un de ses officiers, le maréchal Berthier, qui devient ainsi prince de Neuchâtel. En 1814, la principauté retourne en mains prussiennes lors du traité de Vienne, en même temps qu'elle est intégrée à la Confédération helvétique. Dès lors, le pays jouit du double statut de principauté et de canton souverain de la Confédération des XXII cantons. ${ }^{1}$ En 1848 , la Révolution neuchâteloise marque la fin de l'appartenance à la monarchie prussienne et scelle définitivement le rattachement à la Confédération helvétique. Neuchâtel est désormais officiellement République et Canton. ${ }^{2}$

Une particularité de cette principauté est d'avoir conservé une coutume orale durant la totalité de l'Ancien Régime. Plusieurs projets de rédaction et même de codification des coutumes ont vu le jour, à l'instar de celui du coutumier Hory de 1618, mais aucune de ces entreprises n'a abouti. ${ }^{3}$ Le juriste Samuel Ostervald a même rencontré de grandes difficultés à faire imprimer un coutumier pourtant purement privé.

Outre cette résistance à la mise par écrit du droit, il semble également avoir existé à Neuchâtel un rejet catégorique du droit romain, au profit de la coutume seule. En cas de lacune dans la coutume, la réponse du Conseil étroit consiste généralement en un renvoi auprès des juges censés délibérer dans l'esprit de la coutume. C'est du moins ce qu'affirme Samuel Ostervald, juriste du XVIIIe, lorsqu'il écrit dans son coutumier : "Que nos loix \& nos coutumes l'emportent sur les décisions des loix romaines. " ${ }^{4}$ La même affirmation fait l'objet d'un petit fascicule du disciple de Savigny et premier professeur de droit de l'Université de Neuchâtel, Georges AugusteMatile. ${ }^{5}$ Ces avis concordent avec les déclarations émanant du Petit Conseil de Ville, garant de la coutume neuchâteloise et donc compétent pour définir le droit applicable. Certains historiens du droit tels que JeanFrançois Poudret, ${ }^{6}$ Carlo Augusto Cannata ${ }^{7}$ ou plus récemment Jean-Philippe Dunand ${ }^{8}$ ont nuancé cette affirmation et évoqué une influence du droit romain sans pour autant en apporter la preuve grâce à une étude exhaustive des textes.

\footnotetext{
${ }^{1}$ Confédération des XIX cantons qui devient celle des XXII cantons avec le rattachement de Neuchâtel, du Valais et de Genève.

2 BARTOLINI 2017.

${ }^{3}$ Voir WYSSBROD 2019.

${ }^{4}$ OSTERVALD 1785, p. 354.

5 MATILE 1838.

${ }^{6}$ POUDRET 2002.

${ }^{7}$ CANNATA 1987.

${ }^{8}$ DunAND 2002.
} 
Cet article constitue une étude préliminaire de quelques aspects du droit successoral testamentaire dans la coutume d'époque moderne de la bourgeoisie de Neuchâtel, dont l'une des caractéristiques semble être une transmission particulière du droit romain. La coutume régulant le testament à Neuchâtel à l'époque moderne, et en particulier le nombre de témoins requis, présente en effet certaines caractéristiques formelles identifiables à une surprenante tradition du droit romain. Il s'agit d'explorer l'articulation de ce rapport - réel ou imaginé entre cette coutume et les différentes traditions de droits romains qui ont successivement eu cours en Occident.

Le corpus de cette étude est constitué de deux volumes in-folio ${ }^{9}$ comportant 383 déclarations pour un total d'environ 1500 points ${ }^{10}$ sur près de 850 pages. La totalité de ces déclarations a été transcrite en $\mathrm{XML}^{11}$ selon la version P5 des normes TEI. ${ }^{12}$ La totalité du corpus, ainsi que des photos haute définition des sources, est disponible gratuitement en ligne sur le portail des SDS. ${ }^{13}$ Les textes ne sont pas uniquement transcrits, mais également analysés et balisés. Ainsi des mots clefs ont été définis manuellement après la transcription et l'analyse du texte. Cela permet de trouver rapidement les textes correspondants aux concepts recherchés en éliminant les résultats parasites (bruit documentaire) qui résulteraient d'une recherche en plein texte.

Les résultats peuvent également être restreints en fonction de différents critères tels que la date, le lieu, etc. De même, ils peuvent être élargis à des unités éditoriales d'autres cantons, voir la totalité des textes existants en version numérique augmentée, permettant ainsi des comparaisons entre différentes régions. Les résumés permettent également de savoir rapidement si un résultat est pertinent ou non et les commentaires fourniront bientôt une bibliographie des études dans lesquelles les sources en questions sont citées. Le gain de temps pour les chercheurs est ainsi considérable et permet d'effectuer rapidement des recherches complexes et très précises. Le fac-similé du document permet enfin de revenir à la source lorsque cela est nécessaire, sans avoir à aller en archives.

Les déclarations affirmant que la coutume est la source prioritaire dans le pays de Neuchâtel ont par exemple été balisées avec le concept "primauté de la coutume» (identifiant key005216). ${ }^{14}$ Grâce à cette balise, il est possible de trouver immédiatement les trois points confirmant cette primauté et rejetant le droit romain ${ }^{15}$, alors qu'une recherche plein texte, avec le terme " coutume » fournit pas moins de 261 résultats, et l'expression précise " primauté de la coutume » aucun, car il n'existe pas dans les sources une formule précise pour affirmer cette primauté. Le concept " primauté de la coutume » est par ailleurs lié à celui « droit romain " (identifiant $\underline{\text { key005125 }})^{16}$ permettant de retrouver également un quatrième point de coutume plus tardif, dans lequel il est demandé si le droit romain possède un caractère supplétif en cas de silence de la coutume, ce à quoi le Petit Conseil refuse de répondre, renvoyant à une décision de justice. ${ }^{17}$

\section{Degré de romanité de la coutume neuchâteloise}

Au sein du corpus que représente l'édition en ligne des points de coutume de Neuchâtel, l'une des déclarations les plus parlantes pour l'étude du droit successoral est celle du 8 mai $1705 .{ }^{18}$ Celle-ci renferme de nombreux détails sur la forme que doit revêtir un testament à Neuchâtel. Elle suit la forme générale des autres déclarations de la coutume, soit d'abord une succession de demandes formulées par le requérant et numérotées. Les deux premières questions concernent les sources du droit et précisément la validité du droit romain à Neuchâtel, ainsi que la nature de la coutume neuchâteloise :

\section{Si dans la souveraineté de Neufchatel l'on juge suivant le droit romain ou suivant une coutume locale?}

\footnotetext{
${ }^{9}$ CH NE AVN B 101.14.001 et CH NE AVN B 101.14.002.

${ }^{10}$ La plupart des déclarations, rendues à la demande des justiciables, avocats ou juges, comportent plusieurs points de coutumes distincts.

11 «Extensible Markup Language», en français « langage de balisage extensible ».

${ }^{12}$ «Text Encoding Initiative», en français « initiative pour l'encodage du texte », voir https://tei-c.org/guidelines/p5/.

${ }^{13}$ https://www.ssrq-sds-fds.ch/exist/apps/ssrq/.

${ }^{14}$ https://www.ssrq-sds-fds.ch/lemma-db-edit/views/view-keyword.xq?id=key005216.

${ }_{15}$ SDS NE $3 \underline{16731029} ; \underline{17050508} ; \underline{17060211}$. (pour la cote complète et l'URL, voir " sources primaires » en fin d'article).

${ }^{16} \mathrm{https}$ ///www.ssrq-sds-fds.ch/lemma-db-edit/views/view-keyword.xq?id=key005125.

17 SDS NE $3 \underline{17970206 .}$

${ }^{18}$ SDS NE $3 \underline{17050508}$, dont la transcription est reproduite intégralement en annexe ci-dessous.
} 


\section{Si par la coutume de Neufchastel, l'on n'entend pas tant les anciennes usances qui ne sont point redigées par ecrit, que les declarations rendues par messieurs du Conseil Etroit de cette ville et qui se trouvent par ecrit scelées du seau de la ville et signées par le secrétaire de ville, ou vidimées et collationnées sur l'original par un notaire fameux?}

Une fois que le particulier a terminé la liste de ces demandes, les déclarations de coutume font généralement état de la tenue de délibérations au sein du Conseil étroit. Celle du 8 mai 1705 ne fait pas exception. Les réponses sont ensuite données en suivant la même numérotation, non sans quelques libertés :

Mesdits sieurs du Conseil, ayant eu avis par ensemble, donnent par declaration, que de tout tems immemorial de pere à fils jusqu'a present, la coutume de Neufchastel est telle.

Assavoir, sur le 1. et 2. article que, en jugeant, on ne suit pas le droit romain, mais bien la coutume particuliere et ancienne ecrite et non ecrite usitée dans cette souveraineté.

Malgré la tentative de définir ou plutôt de circonscrire exactement la nature de la coutume neuchâteloise de la part du demandeur, le Conseil étroit se contente de botter en touche et de donner une réponse générale. Ainsi, dans cette déclaration du 8 mai 1705, le Conseil étroit réalise deux choses : il repousse d'abord l'autorité du droit romain, mais il frustre aussi d'emblée les velléités de circonscrire la coutume à certains types de sources du droit. Ainsi, il est possible d'avancer que le rejet du droit romain ne porte en réalité pas sur son contenu, mais bien sur ce qu'il représente : un droit savant. Nous y revenons à la fin de cet article. Retenons pour le moment que le rejet du droit romain revient régulièrement dans les points de coutume neuchâtelois. On retrouve ainsi des variantes de la phrase « En jugeant l'on ne suit pas le droit romain, mais la coutume particulière et ancienne qui a été pratiquée et usitée dans cette souveraineté " dans trois des points de coutumes du corpus. ${ }^{19}$ Une déclaration du 6 février 1797 refuse de répondre à la nature supplétive du droit romain et renvoie à une connaissance de justice. ${ }^{20}$

Pourtant, la nature du droit successoral testamentaire de la coutume neuchâteloise offre des formes dont certaines rappellent le droit romain. En particulier Jean-François Poudret, dans son étude des coutumes des différents cantons et régions de Suisse, avait déjà brièvement souligné l'usage dans le testament en terres neuchâteloises de l'institution d'héritier et de certaines caractéristiques formelles en accord avec le droit romain. ${ }^{21}$ Il est par ailleurs connu que le droit successoral occidental, en particulier testamentaire, entretient une dette importante vis-à-vis du droit romain. La succession testamentaire est en effet historiquement caractérisée par un important formalisme, lequel vise à garantir la solennité et l'authenticité des décisions prises par la personne décédée. ${ }^{22}$

L'usage de testaments est ancien dans la région de Neuchâtel. On sait que ce droit est conféré aux bourgeois de la ville par la Charte de franchise de $1214 .^{23}$ Les héritiers bourgeois échappent ainsi à la mainmorte et en plus de cela la capacité testamentaire est accordée et libre de droits de regard du seigneur, sauf si les bénéficiaires devaient compter parmi les rangs des moines cisterciens. En voici une traduction :

Et si aucun des bourgeois meurt, ses héritiers lui succéderont pleinement en l'héritage sans reprise, toutefois ils doivent le recevoir de la main du seigneur.

\footnotetext{
19 SDS NE $3 \underline{16731029} ; \underline{17050508 ;} ; 17060211$.

20 SDS NE $3 \underline{\underline{17970206}}$.

${ }^{21}$ Voir MATILE 1838, POUDRET 2002, DUNAND 2002.

22 Voir LAURENT-BONNE 2017.

${ }^{23}$ Cette charte fut faite " selon la coutume de Besançon " et des liens juridiques persistent avec cette ville jusqu'au XVII ${ }^{\mathrm{e}}$ siècle. Du moins Neuchâtel reste-t-elle aux us et coutume de Besançon au moins jusqu'en 1530 . En cas de problème, les juges neuchâtelois pouvaient alors s'y rendre pour prendre conseil " aux entrèves ». Seuls quatre recours aux entrèves sont attestés, en 1402, 1455, 1473 et 1530. En 1623 enfin, c'est également à Besançon que le coutumier Hory est envoyé pour être revu et corrigé par le docteur Claude-Antoine Buson, conseiller de Besançon. Voir FAVARGER 1967, p. 64 et 70 ; MOREROD/SCHEURER 2017. Besançon a conservé pendant longtemps le plus important manuscrit du Bréviaire d'Alaric, le Vesontinus, édité par Cujas en 1566. Si cette tradition a pu exercer une quelconque influence sur la coutume bysontine, c'est peut-être par ce biais que du droit issu du Bréviaire a pu s'insinuer dans la coutume neuchâteloise.
} 
Aussi, ils peuvent faire un testament de leurs possessions sans notre consentement, excepté en faveur des moines blancs, réservé notre droit. ${ }^{24}$

Ce texte continue sans doute de constituer une source ou du moins d'exercer son influence sur la tradition de la coutume bourgeoise à la période moderne. Ainsi un point de coutume de la déclaration du 8 mai 1705 rendu par le Conseil Étroit préserve une formulation étonnamment proche qui assure la capacité testamentaire aux individus de franche condition :

\section{Sur le 8. Toute personne qui est de franche condition et qui n'a point d'enfans peut tester} et disposer de tous ses biens et les donner à qui il lui plait, excepté à moines blancs.

On retrouve ainsi la référence aux moines blancs, devenue saugrenue dans la mesure où le pays est alors passé à la Réforme depuis près de deux siècles et que la présence cistercienne s'est drastiquement effacée. Ce particularisme montre cependant une continuité entre la coutume moderne et les franchises bourgeoises accordées au XIII siècle par les comtes de Neuchâtel.

Le testament de coutume neuchâtelois, tel qu'il est décrit dans le point de coutume en annexe relève essentiellement du droit coutumier. Pour ne citer qu'un exemple des caractéristiques des testaments coutumiers, le testament neuchâtelois demande que les plus proches parents, héritiers ab intestat s'il n'y a pas d'enfants légitimes, soient exhérédés nommément dans un testament et que dans ce cas il leur soit légué au minimum 5 sols. C'est là un particularisme local qui s'observe dans notre déclaration du 8 mai 1705 :

Sur le 4. 5. et 6. Celui, (n'ayant point d'enfans) qui veut exhereder de ses biens aucuns de ses plus proches parens, lesquels à deffaut d'enfans legitimes devroient estre selon le droit de nature ses heritiers legitimes et ab intestat s'il n'en estoit disposé autrement, il les doit specifiquement nommer, et declarer ce qu'il lègue et ordonne à un chacun d'eux en département de ses biens, l'or, argent, obligations, terres ou autres choses et pour le moins la valeur de cinq sols foibles.

Nous ne pouvons pas nous livrer ici à une étude exhaustive des caractéristiques du testament de droit coutumier neuchâtelois, et nous préférons plutôt nous concentrer sur deux aspects particuliers qui sont probablement les plus fortement influencés par le droit romain : d'abord l'institution d'héritier, et surtout le nombre de témoins requis. Ces deux brèves études de cas devraient permettre une intéressante mise en relation avec un problème historique du droit romain.

L'institution d'héritier, tout d'abord, est une caractéristique importante du testament de droit romain classique : I'héritier est universel et succède au de cujus dans ses droits et devoirs, il est notamment responsable d'honorer les dettes, mais aussi les legs ordonnés par le défunt. Concernant les documents de la pratique médiévale, Georges-Auguste Matile a publié au XIXe siècle déjà une série de testaments de la région de Neuchâtel que JeanFrançois Poudret analyse brièvement. ${ }^{25}$ Le premier testament qui présente des caractéristiques du droit romain conservé à Neuchâtel est celui du chanoine Henri de Cormondrèche en 1281 avec institution d'héritier, clause codicillaire et le sceau de huit témoins. ${ }^{26}$ Le premier testament de laïc conservé date de 1308 et émane d'une bourgeoise de Neuchâtel, Guillemette du Four. Le document se rapproche d'une donatio mortis causa avec deux exécuteurs testamentaires pour des legs, et pas d'institution d'héritier. ${ }^{27} D^{\prime}$ autres exemples de testaments avec institutions d'héritiers sont connus au XIVe siècle, mais avec des irrégularités. Dans l'ensemble Jean-François Poudret remarque qu'on observe une certaine confusion avec les donations jusqu'au XV siècle. $^{28}$

\footnotetext{
${ }^{24}$ Charte de 1214 « Si autem aliquis burgensium obierit, heredes sui plene succedent in hereditatem sine replacito, recipere tamen debent de manu domini. Testamentum autem facere possunt de suis possessionibus absque conscientia nostra, salvo tamen jure nostro, preterquam albis monachis. "Selon l'édition GIRARBILLE 2014.

${ }^{25}$ Voir POUDRET 2002, p. 205-207.

${ }^{26}$ MATILE 1844, p. 185ss, n 215. Voir PoudRet 2002, p. 206.

${ }^{27}$ MATILE 1844, p. 269, n' 293.

28 POUDRET 2002, p. 212.
} 
En revanche, notre déclaration du 8 mai 1705 exige clairement la présence d'une institution d'héritier dans le testament coutumier neuchâtelois et il en va de même dans une série de points de coutume depuis $1664 .{ }^{29}$ Voici le point de 1705 :

Sur le 7. dans tous les testamens il y doit avoir institution d'heritier, et celui qui en consequence se porte heritier d'un defunt est obligé d'acquitter les dettes et les legs dudit defunt. ${ }^{30}$

L'influence du droit romain est ici probablement à imputer aux juristes, selon une tradition savante. Ainsi, dans le Coutumier Hory, réalisé entre 1610 et 1618 à Neuchâtel, il est indiqué :
Les formalitez d'un testament requises selon la coustume de ceste souveraineté et qui s'observent, sont telles qu'il faut qu'il y soit appellé avec un notaire fameux non moings de quatre tesmoings, personnes virilles, gens d'honneur, bien cogneuz de ceux du lieu, \& qui ne soyent domestiques ny parents du testateur ny moings du notaire, \& non forcez a ce tesmoignage : Et que le testateur institue un heritier, car le chef \& vray fondement de tous testaments, c'est l'institution d'heritier.

Cette phrase "le testateur institue un héritier, car le chef et fondement de tous vrais Testamens c'est l'institution d'héritier » se reconnaît comme une traduction presque littérale d'un passage des Institutes de Gaius, aussi cité par Ulpien et repris dans les Institutes de Justinien. ${ }^{31}$ Malgré cette possible influence savante, Poudret remarque que, dans la pratique, l'institution d'héritier n'est pas forcément bien comprise au point de vue technique dans les pays romands. Les héritiers institués sont parfois les héritiers naturels sans autres dispositions, ce qui n'a aucun sens en droit romain, ou des catégories d'individus vagues, des inconnus comme "les pauvres " par exemple, alors que les testaments en faveur d'incertae personae n'étaient pas valides selon le droit justinien. ${ }^{32}$

Il faut aussi remarquer le Coutumier Hory constitue en réalité une tentative avortée de rationaliser la coutume neuchâteloise et que son influence aura probablement été nulle en ce qui concerne le nombre de témoins requis pour assurer la validité d'un testament. ${ }^{33}$ Hory fixait en effet ce certus numerus à quatre, une situation qui entre en contradiction avec une précédente décrétale du 25 octobre 1537. Par le biais de celle-ci, les Audiences générales de Neuchâtel ont fixé le nombre de témoins à " sept ou cinq ", et cela va servir de source à la coutume neuchâteloise dans plusieurs déclarations. À cette période, les Audiences possèdent encore le pouvoir législatif, qui sera récupéré au début du XVII e siècle par le Tribunal des Trois-États. Les cantons combourgeois y sont indirectement représentés en raison de droits que possèdent certains Bernois et Soleurois à Neuchâtel en vertu de leurs titres. Les Audiences défendent ainsi certains acquis issus de la période où Neuchâtel était sous l'administration des Confédérés en raison d'un arrêt d'interdiction prononcé par François ler contre Jeanne de Hochberg. Les Audiences font alors preuve d'une plus grande acceptation des droits savants, y voyant probablement un moyen d'enrayer une dégradation de la justice depuis la fin du régime d'occupation par les cantons helvétiques. En 1532 ce corps soutient par exemple une demande de codification, émanant des Confédérés ou peut-être même des Bernois seuls, elle aussi avortée. ${ }^{34}$

Voici le texte de la décrétale du 25 octobre 1537 qui se prononce sur le nombre de témoins :

\footnotetext{
${ }^{29}$ SDS NE $3 \underline{16640517} ; \underline{16690504} ; \underline{16960228 \mathrm{~b}} ; \underline{17050508} ; \underline{17230306}$.

30 SDS NE $3 \underline{17050508 .}$

${ }^{31}$ Gai. Inst. 2, 229 " Ante heredis institutionem inutiliter legatur, scilicet quia testamenta vim ex institutione heredis accipiunt; et ob id velut caput et fundamentum intellegitur totius testamenti heredis institutio. " lust. Inst. 2, 20, 34 " Ante heredis institutionem inutiliter antea legabatur, scilicet quia testamenta vim ex institutione heredum accipiunt et ob id veluti caput atque fundamentum intellegitur totius testamenti heredis institutio. "

32 POUDRET 2002, p. 553.

${ }^{33}$ Demandé en 1610 par Catherine de Gonzague, assurant la tutelle pour son fils Henri II d'Orléans-Longueville, le coutumier Hory est un ouvrage remarquable. Cette tentative de synthèse du droit neuchâtelois en 59 articles présente des caractéristiques étonnantes et apparaît tout à fait en avance sur son temps en regard des autres coutumiers. Terminé en 1618, il se heurta à la résistance du Petit Conseil. II semble être abandonné vers 1626, après une mise à l'essai dans les juridictions de La Chaux-de-Fonds et de La Brévine, mais des copies sont encore réalisées tardivement et circulent à Neuchâtel, mais également à Berne. Voir WYSSBROD 2019, p. 58-62.

${ }^{34}$ Voir WYSSBROD 2019, p. 54-56.
} 


\begin{abstract}
I'on desfend a tous clercz notayres dudict comté qu'ilz ne reçoipvent testamentz ny donnations entre les vifs que pour les moins ilz n'y appellent sept ou cinq tesmoins non suspectz a peyne d'estre privez de son estat et office. Sauf et reservé en cas de necessité. ${ }^{35}$
\end{abstract}

Ce nombre de témoins « sept ou cinq » a retenu toute notre attention, car il s'agit potentiellement d'un élément particulier du droit romain des successions. Ainsi, au printemps 2019, lorsque le portail comportait 383 déclarations de la coutume, ${ }^{36}$ interroger le moteur de recherche des Sources du Droit Suisse donnait huit résultats où les points de coutume de Neuchâtel mentionnent que le nombre de témoins requis pour un testament est de " cinq à sept $"{ }^{37}$ Étrangeté, ambivalence, dualisme ou illogisme ? À cet égard, le point de coutume du 8 mai 1705 n 13 sous-entendent que cinq témoins suffisent en réalité pour qu'un testament soit valide.

Sur le 13. Il n'est pas necessaire qu'un testament passé par main de notaire en presence de cinq témoins non suspects soit signé du testateur ni des témoins. ${ }^{38}$

Cette hésitation sur le nombre de témoins semble devoir être rapprochée d'une phase de l'évolution historique du testament de droit romain appelée «dualisme » par la recherche. Pour revenir brièvement sur les métamorphoses que subit l'institution du testament de droit romain, il faut se rappeler que le testament de droit classique, per aes et libram, implique la performance d'abord réelle puis symbolique d'une mancipatio par nuncupatio, soit une vente fictive énoncée oralement et qui requiert la présence de témoins pour être valide. Au niveau performatif, sept individus et le testateur sont en réalité impliqués. Parmi ceux-ci on compte cinq témoins, auxquels s'ajoutent l'acheteur fictif (le familiae emptor) et le libripens chargé de vérifier symboliquement le poids de la pièce en cuivre. ${ }^{39}$ Par la suite, alors même que la mancipatio est devenue désuète et que le testament assume sa forme écrite, on continue d'exiger cinq témoins pour la validité d'un testament de droit civil. ${ }^{40}$ En revanche en droit prétorien, les documents nécessaires pour permettre l'octroi de la bonorum possessio requièrent la signature de sept témoins. En droit classique le testament prétorien n'existe pas en tant quel tel, il s'agit d'un document - souvent un testament invalide en droit civil pour des raisons formelles - qui va produire des résultats comparables. Cette distinction va s'effacer en droit postclassique et un testament de droit prétorien est fréquemment mentionné dans les sources à partir du IVe siècle, lequel requiert la présence de sept témoins. ${ }^{41}$ L'idée même d'un testament prétorien à sept témoins est ainsi une construction du droit postclassique et préjustinien. En effet, au moment où Justinien promulgue le Corpus luris Civilis entre 529 et 534, le nombre de sept témoins est requis pour le testament de droit romain dit tripartitum, nous allons y revenir.

C'est en effet à ce niveau-là que le problème des sources du droit coutumier neuchâtelois se complexifie. En effet, toutes les mentions conservées d'une différence faite entre les testaments de cinq ou à sept témoins se concentrent dans le Code Théodosien ${ }^{42}$ : CTh. 4.4 .1 (326 Constantin) ${ }^{43}$; CTh. 4.4.7.2 (424 Theodose II) ${ }^{44}$ et ses Interpretationes (4.4.3 interpretatio d'une constitution des empereurs Arcadius et Honorius, probablement de 396). ${ }^{45}$ Ces interpretationes ont été éditées par Mommsen au bas des constitutions impériales, mais elles sont en réalité tirées d'une œuvre bien différente, soit la Lex Romana Visigothorum ou Bréviaire d'Alaric, promulgué

\footnotetext{
${ }^{35}$ Cité d'après FAVARGER/TRIBOLET 1982 nº 67, p. 168.

${ }^{36}$ Une centaine de déclarations (ou parfois le refus ou l'impossibilité d'en donner une) tirés des Manuels de conseil de Ville vont encore compléter ce corpus, d'ici l'été 2020.

${ }^{37}$ SDS NE $3 \underline{16620507} ; \underline{16580326} ; \underline{16800121} ; \underline{16810223} ; \underline{16810621} ; \underline{16960228 \mathrm{~B} ; \underline{17220420} .}$. En revanche, SDS NE $3 \underline{17041205}$ mentionne cinq témoins.

${ }^{38}$ SDS NE $3 \underline{17050508 .}$

${ }^{39}$ Ainsi on obtient dans cette configuration sept individus, mais cinq témoins à proprement parler, ce qui constitue possiblement l'origine du nombre de sept témoins.

${ }^{40}$ La réforme des formules testamentaires prend vraisemblablement place sous Constantin et répond à une désuétude déjà consommée de la mancipatio. Cf. C. 6.23.15.0. Voir NOWAK 2011.

${ }^{41}$ Voir ARCHI 1981 ; LAURENT-BONNE 2017, p. 24-25.

${ }^{42}$ Ajoutons Isid. Or. 5.24 : « Testamentum iuris civilis est quinque testium subscriptione firmatum. Testamentum iuris praetorii est septem testium signis signatus. "

${ }^{43}$ CTh. 4.4.1 « In codicillis, quos testamentum non praecedit, sicut in voluntatibus testamenti septem testium vel quinque interventum non deesse oportet ».

${ }^{44}$ CTh. 4.4.7.2 « quod quidem nullam habeat firmitatem, nisi aut septem aut quinque vel rogati aut qui fortuitu venerint, possint iure testimonium perhibere ".

${ }^{45}$ CTh. 4.4.3.3 Interpr. « Si moriens, cum scribit aut dictat chartulam testamenti, praetermiserit forsitan vocabulum ponere, aut civilis, id est, quod quinque testium, aut praetorii iuris, quod septem testium erit subscriptione firmandum ».
} 
dans le royaume wisigoth par le roi Alaric II en 506 et qui a préservé les passages correspondants du Code Théodosien par ailleurs perdus.

Ces interpretationes paraissent avoir été rédigées expressément pour la compilation de la Lex Romana Visigothorum ou du moins avoir été adaptées à ce moment-là. ${ }^{46}$ Elles sont ainsi probablement représentatives d'une tradition juridique ou scolaire gauloise de la fin du Ve siècle par ailleurs mal connue. L'interpretatio 4.4.3 explique du reste l'origine historique du dualisme testamentaire en établissant clairement la différence entre les testaments de droit civil à cinq témoins et ceux de droit prétorien à sept témoins. Dans la Lex Romana Burgundionum 45.2 cette dualité est aussi présente, ${ }^{47}$ de même que dans l'Edictum Theodorici $28 .{ }^{48}$

Pourtant, l'empereur d'Orient Théodose II avait précédemment réformé en profondeur le système de la succession testamentaire. Dans sa Novelle datée de 439,49 soit plusieurs décennies avant la compilation wisigothique, Théodose II ne fait pas mention du dualisme, il ne parle que de sept témoins et interdit notamment qu'un testament nuncupatif (oral) se fasse devant moins de sept témoins. On notera en revanche que l'interpretatio présente dans l'édition de Mommsen - mais plus tardive et de tradition occidentale comme on l'a mentionné - s'empresse quant à elle de rétablir une différence entre testament de droit civil et testament de droit prétorien. De même, on remarquera aussi que la Novelle 21 de Valentinien, empereur d'Occident, promulguée en 446 mentionne bien le dualisme. ${ }^{50}$

L'importance accordée à ce dualisme est ainsi probablement un trait caractéristique du droit postclassique et peut-être bien de la tradition occidentale, en particulier de la culture juridique absorbée par les codes des nouveaux royaumes. Celle-ci aura résisté à la réforme de la succession testamentaire promulguée par Théodose en 439 dans sa Novelle et appliquée par la partie orientale de l’Empire. Par contraste encore, les Institutes de Justinien de 533, rappellent que les différences entre testaments de droit civil et de droit prétorien ont cessé d'exister tant par la pratique que par les édits des empereurs, et qu'un testament - désormais une synthèse des différents droits, d'où son nom de testamentum tripartitum - requiert la présence de sept témoins. ${ }^{51} \|$ est difficile de reconstituer cette tradition du droit occidental en détail, car celui-ci n'a survécu que dans les interprétations wisigothiques et quelques autres manuscrits. Essayer de résoudre ce puzzle a suscité une littérature non négligeable et cela dépasse totalement notre propos. ${ }^{52}$ Mentionnons seulement que l'hypothèse la plus raisonnable émane de Max Kaser, lequel pensait qu'il s'agissait d'une Formularpraxis. ${ }^{53}$

La seule survivance éventuelle du dualisme testamentaire encore en application dans l'œuvre de Justinien réside dans une éventuelle exception pour les endroits reculés, émise en 534. L'empereur prévoit ainsi que l'on puisse abaisser le nombre de témoins à cinq s'il était impossible d'en rassembler sept. ${ }^{54}$ Deux autres textes font

\footnotetext{
${ }^{46}$ Voir notamment MATTHEWS 2001.

${ }^{47} L R B 45.2$ « Si vero testis adhibentur, ante quos testator vel suscribat vel suum testamentum offerat subscribendum, cives Romani testis adhibendi sunt, numero conpetenti, vel $\mathrm{V}$ vel vii, qui ad testamentum iure adhiberi possunt quorumque testimonium recipetur, cum quibus est nobis testamenti factio, secundum legem Theudosii et Valentiniani ad Florentium praefectum preturii datam. " « Si l'on fait participer des témoins, le testateur écrira ou fera écrire son testament ; les témoins seront choisis parmi les citoyens romains. lls devront être au nombre de cinq et jusqu'à sept, et attester qu'ils ont bien compris les termes du testament et qu'ils les confirment, selon la loi de Théodose et de Valentinien, donnée par Florent, le préfet du prétoire. " Voir SANCHEZ-MORENO p. 248-249.

${ }^{48} \mathrm{Ed}$. Theod. 28 " Faciendorum testamentorum omnibus, quos testari leges permittunt, damus late licentiam: ita ut septem aut quinque testes ingenui ac puberes in conspectu testatoris, uno tempore, eodem rogante subscribant. »

${ }^{49}$ Nov. Theod. 16.

${ }^{50}$ Nov. Val. 21.1.4. «Idcirco, quia minutiis priscae consuetudinis et obscuritate submota solam defunctorum convenit inspici voluntatem, cui multum roboris erit si vel septem vel quinque testibus muniatur, et virum spectabilem leonium praeter fiduciam precum pridem cum uxore communium voluntas quoque alia et subscriptionibus testium munita defendit, apud eundem solida successione iucundae coniugis suae iugiter permanente, quisquis maluerit delata nobis supplicatione testari habeat liberam facultatem. "

${ }^{51}$ Inst. Just. 2.10.3 « Sed cum paulatim tam ex usu hominum quam ex constitutionum emendationibus coepit in unam consonantiam ius civile et praetorium iungi, constitutum est, ut uno eodemque tempore, quod ius civile quodammodo exigebat, septem testibus adhibitis et subscriptione testium, quod ex constitutionibus inventum est, et ex edicto praetoris signacula testamentis imponerentur: ut hoc ius tripertitum esse videatur, ut testes quidem et eorum praesentia uno contextu testamenti celebrandi gratia a iure civili descendant, subscriptiones autem testatoris et testium ex sacrarum constitutionum observatione adhibeantur, signacula autem et numerus testium ex edicto praetoris. "

52 On prendra soin d'attendre les résultats du projet $\underline{\text { REDHIS. }}$

53 KASER 1959, p. 479.

${ }^{54}$ C. 6.23 .31 .3 « Si autem in illo loco minime inventi fuerint septem testes, usque ad quinque modis omnibus testes adhiberi iubemus: minus autem nullo modo concedimus. "
} 
référence à des actes de libéralités pour cause de mort non testamentaires et prévoient cinq témoins. ${ }^{55}$ ॥ est possible que cela ait laissé des traces dans la tradition médiévale du droit romain, mais généralement lié à l'exception d'un cadre rustique dont il n'est jamais fait mention dans la coutume neuchâteloise. La coutume neuchâteloise se trouve dans une tradition coutumière bien éloignée du droit canon ou d'autres coutumes médiévales de différentes régions de France où deux témoins sont généralement suffisants en plus du notaire. ${ }^{56}$ En revanche, il est difficile de saisir d'où provient l'influence de cette tradition particulière du droit romain, celle du dualisme du testament, qui prévoit de manière conservatrice la présence de cinq ou sept témoins, selon que la tradition soit celle du droit civil ou du droit prétorien.

Quant à identifier la relation entre le droit romain et la coutume neuchâteloise, il semble possible d'avancer que le rejet du droit romain est bien plus symbolique que réel. Cette vision est exprimée par Ostervald, qui reconnaît timidement le recours au droit romain de manière supplétive, pas en tant que droit romain, mais uniquement, car il s'agit d'une décision juste, la meilleure qui soit.

«Il semble à bien des gens que cette déclaration interdit aux juges tout égard \& toute attention pour les décisions des loix Romaines, \& que ces décisions, quand elles seroient les plus justes du monde, doivent être rejetées, même au défaut de nos Coutumes, par la seule raison qu'elles sont tirées des loix Romaines : sentiment absurde, s'il en fut jamais, auquel pourtant les préjugés, joints à l'ignorance, ont donné crédit chez bien des gens.

Voici quel est le véritable sens de cette déclaration :

1 o. Que dans les jugemens on suit la coutume particuliere \& ancienne du pays, \& non pas les loix Romaines, qui n'ont pas force de loi dans cet Etat, parce qu'elles n'y ont jamais été reçues ni adoptées.

2 o. Que lorsque nos Coutumes sont en opposition avec les loix Romaines, il est incontestable qu'elles doivent l'emporter sur ces dernieres.

Voilà tout ce qu'on peut raisonnablement insérer de la déclaration ; mais il n'est pas permis d'en conclure que, dans le cas où nos usages gardent le silence $\&$ ne disent mot, il faille rejeter les décisions du droit Romain, quoiqu'on les trouves trèsjustes \& très-équitables, par la seule raison qu'on les a puisées dans le corps du droit Romain, Il faut saisir la justice \& l'équité par-tout où on a le bonheur de les trouver, \& se conformer aux loix Romaines dans ce cas, \& même à toutes autres ; non pas parce qu'elles sont loix Romaines, mais par la seule raison que leurs décisions sont justes, \& qu'on n'a rien trouvé de meilleur ailleurs. » $\$ 57$

\section{Conclusion}

II semble ainsi possible d'avancer que les autorités neuchâteloises ne s'opposent pas entièrement au droit romain qui, comme on le constate avec les successions testamentaires, en a pénétré la coutume, mais bien plutôt à une conception savante du droit. Cette crainte du droit savant et donc du droit romain trouve probablement son origine dans le fonctionnement politique de la principauté et en particulier dans le caractère étranger du prince. Cette opposition peut être vue comme l'expression d'une volonté d'éviter une appropriation du droit par le prince, ou de reconnaître de manière générale l'autorité d'experts externes à la principauté. En revanche, lorsque des éléments de droit romain, comme le nombre de témoins requis pour la validité d'un testament, ont été intégrés à la coutume, ce droit est parfaitement accepté dans la mesure où il est considéré comme partie intégrante de la coutume et non plus comme du droit savant, quelles que soient ses origines. Le rejet du droit romain lié à des motifs souverainistes mériterait ainsi sa propre histoire.

Si des études de cas ultérieures mériteraient d'être menées, cet article a mis en évidence plusieurs éléments de la succession testamentaire neuchâteloise qui peuvent être mis en relation avec des formes issues du droit romain. L'institution d'héritier est ainsi formellement requise. Bien que soutenue par la norme, la pratique est cependant mal appliquée. L'institution n'est pas suffisamment distinctive pour en suivre l'origine, celle-ci provient peut-être du droit canon, lui-même influencé par le droit romain. Plus probante pour se rendre compte du degré de romanité de cette coutume est l'exigence d'un certus numerus pour les témoins en succession testamentaire. Ainsi, le nombre de témoins nécessaires à la confection d'un testament est fixé à " cinq à sept " par la Décrétale de 1537. On peut y percevoir l'influence formulaire d'un droit romain qui ne correspond lui-

\footnotetext{
${ }^{55}$ C. 6.36.8.3 (Théodose) dernière volontés excepto testamento ; C. 8.56(57).4.0 (Justinien 530 EC) : donatio mortis causa. Sur la question de l'exigence d'un certus numerus dans les actes médiévaux voir MAUSEN 2006, surtout p. 705.

${ }^{56}$ Voir MAUSEN 2006, p. 703-707 ; LAURENT-BONNE 2017, p. 36-40.

${ }^{57}$ OSTERVALD 1785, p. XIV-XV.
} 
même pas au droit romain classique, ni même au droit romain oriental postclassique après la réforme de 439, mais bien à une tradition qui reconnaît un dualisme historique des formes testamentaires, probablement issue de la pratique ou des écoles locales du droit occidental.

La question de savoir quelle source a pu pousser les Audiences générales de Neuchâtel à arrêter le nombre de témoins requis à cinq ou sept, arrimant la coutume neuchâteloise à une tradition de droit romain pourtant éloignée de celle retenue par les pandectes et enseignée à l'époque moderne, demeure donc ouverte. Si l'on peut probablement exclure une filiation directe entre le droit wisigothique et la coutume neuchâteloise, il reste à identifier la tradition médiévale héritée de la tradition romaine occidentale. II apparaît ainsi intéressant de se rendre compte qu'avant de parler d'influence du droit romain ou de degré de conformité au droit romain d'un ordre juridique, il convient de se demander de quelle tradition de droit romain on traite.

\section{Bibliographie sélective}

ARCHI Gian Gualberto, "Testamentum civile, testamentum praetorium », in : Scritti di diritto romano II, Milan 1981, p. 771-808.

BARTOLINI Lionel et al., « Neuchâtel (canton) », in : Dictionnaire historique de la Suisse (DHS), version du 30.05.2017.

BAUER-GERLAND Friederike, Das Erbrecht der Lex Romana Burgundionum, Berlin 1995.

CANnATA Carlo Augusto, "Le droit écrit à Neuchâtel, pays de coutumes ", in : Pays de Neuchâtel, son identité, son environnement, Neuchâtel 1987.

DUNAND Jean-Philippe, "Le testament oral en droit suisse et dans l'ancien droit neuchâtelois », in : Robert Roth/Jean Kellerhals, Dominique Manal-Wehrl (éd.), Pour un droit pluriel : Études offertes au professeur Jean-François Perrin, Genève 2002, p. 33-58.

Favarger Dominique/Tribolet Maurice de, Les sources du droit du canton de Neuchâtel, t. 1, Aarau 1982.

FAVArger Dominique, "À propos du coutumier Hory de 1618 », in : Musée Neuchâtelois, 1970, p. 69-72.

FAVARGER Dominique, "Coutumes et coutumiers neuchâtelois à la fin de l'Ancien Régime ", in : Musée Neuchâtelois, 1967, p. 60-78.

GIRARDBille Olivier, FUhrer Lorraine, IORI Stefano, La Charte De Franchises De Neuchâtel : Avril 1214-Avril 2014 : $800^{\circ}$ de La Fondation De La Communauté Urbaine De Neuchâtel, Neuchâtel 2014.

KASER Max, Das Römische Privatrecht II : Die nachklassischen Entwicklungen, $2^{\mathrm{e}}$ éd., München 1975.

LAURENT-BONNE Nicolas, "La dictée du testament authentique, un succédané de formalisme rituel ", in : Sandrine TISSEYRE/Nicolas LAURENT-BONNE (dir.) Le formalisme. Sources et technique en droit privé positif, Lextenso, Coll. Contextes Culture du droit, 2017, p. 11-54.

MATILE Georges-Auguste, Déclaration ou points de coutume rendus par le Petit-Conseil de la Ville de Neuchâtel, Neuchâtel 1836.

MATILE Georges-Auguste, De l'autorité du droit romain, de la coutume de Bourgogne et de la Caroline, Neuchâtel 1838.

MATILE Georges-Auguste, Monuments de l'histoire de Neuchâtel, Neuchâtel 1844. Volume 2.

MATTHEWS John, "Interpreting the Interpretationes of the Breviarium », in : Law, Society, and Authority in Late Antiquity, Oxford/New York 2001, p. 11-32.

MAUSEN Yves, Veritatis Adiutor : La procédure du Témoignage dans le droit savant et la pratique française (XII XIVe siècles), Milan 2006.

Morerod Jean-Daniel/SCHEURER Rémy, "Neuchâtel (canton), 2.2 Gouvernement et institutions au Moyen Âge ", in : Dictionnaire historique de la Suisse (DHS), version du 30.05.2017.

OSTERVALd Samuel, Les loix, us et coutumes de la Souveraineté de Neuchâtel et Valangin, Neuchâtel 1785.

PETITJEAN Michel "L'acte à cause de mort dans la France coutumière du Moyen Âge à l'époque moderne ", in : Actes à cause de mort. Europe médiévale et moderne [Recueils de la Société Jean Bodin t. LX], Bruxelles 1993, p. 85-127.

POUDRET Jean-François, Coutumes et coutumiers : Histoire comparative des droits des pays romands du XIIIe à la fin du XVI siècle. T. IV Successions et testaments, Berne 2002.

SÁNCHEZ-Moreno ElLART Carlos, «The Late Roman Law of Inheritance: the Testament of Five or Seven Witnesses», in : Béatrice CASEAU \& Sabine R. HUEBNER (éd.), Inheritance, law and religions in the ancient and mediaeval worlds, Paris 2014, p. 229-257.

VocI Pasquale, "II diritto ereditario romano nell'età del tardo impero : il IV secolo. Prima parte ", in : Iura, XXIX, 1978, p. 17-113. 
Vocı Pasquale, « II diritto ereditario romano nell'età del tardo impero : il IV secolo. Seconda parte ", in : Studi in onore di Cesare Sanfilippo, II. Ed., Milano 1982, p. 657-735.

Vocı Pasquale, «II diritto ereditario romano nell'età del tardo impero : il V secolo ", Studia et Documenta Historiae et luris, XLVIII, 1982, p. 1-125.

WYSSBROD Adrien, De la coutume au code, résistances à la codification du droit civil à Neuchâtel sous l'Ancien Régime, Neuchâtel 2019.

\section{Sources primaires}

Les volumes CH NE AVN B 101.14.001 et CH NE AVN B 101.14.002 ont été utilisés dans leur version numérisée, du projet SDS NE 3, sur le portail de la Fondation des sources du droit suisse. Ils sont nommés dans les notes de bas de page selon leur identifiant (SDS NE 3 AAAMMJJ) et listés ci-dessous avec leur côte complète et l'URL de la transcription sur le portail.

SDS NE $3 \underline{16580326}$, CH NE AVN B 101.14.001, fol. 33v-434r

SDS NE 3 16620507, CH NE AVN B 101.14.001, fol. 451r-451v

SDS NE 3 16640517, CH NE AVN B 101.14.001, fol. 460r

SDS NE 3 16690504, CH NE AVN B 101.14.001, fol. 473r-473v

SDS NE $3 \underline{16800121}$, CH NE AVN B 101.14.001, fol. 523v-524r

SDS NE $3 \underline{16810223}$, CH NE AVN B 101.14.001, fol. 526r-526v

SDS NE 3 16810621, CH NE AVN B 101.14.001, fol. 528v

SDS NE 3 16960228b, CH NE AVN B 101.14.001, fol. 561r-562v

SDS NE $3 \underline{16731029}$, CH NE AVN B 101.14.001, fol. 500v-501r

SDS NE 3 17041205, CH NE AVN B 101.14.001, fol. 600r-600v

SDS NE 3 17050508, CH NE AVN B 101.14.001, fol. 601r-605v

SDS NE 3 17060211, CH NE AVN B 101.14.001, fol. 606v-609v

SDS NE $3 \underline{17220420}$, CH NE AVN B 101.14.002, fol. 26v-27r

SDS NE 3 17230306, CH NE AVN B 101.14.002, fol. 33v-35r

SDS NE $3 \underline{17970206}$, CH NE AVN B 101.14.002, fol. 86v 


\section{Annexes}

1705 - 8 mai, Neuchâtel

Cote : AVN B 101.14.001, fol. 601r-605v

\section{SDS NE $3 \underline{17050508}$}

Touchant les testamens.

Sur la requeste presentée à messieurs le maitre bourgeois et Conseil Etroit de la Ville de Neufchastel par le sieur David Dupaquier, notaire bourgeois de ladite ville, agissant au nom du sieur Jaques Baron, bourgeois de Blaye en Guyenne, tendante aux fins d'avoir declaration de la coutume dudit Neufchastel sur les points et articles suivans.

\section{Si dans la souveraineté de Neufchatel l'on juge suivant le droit romain ou suivant une coutume locale?}

2. Si par la coutume de Neufchastel, l'on n'entend pas tant les anciennes usances qui ne sont point redigées par ecrit, que les declarations rendues par messieurs du Conseil Etroit de cette ville et qui se trouvent par ecrit scelées du seau de la ville et signées par le secrétaire de ville, ou vidimées et collationnées sur l'original par un notaire fameux?

3. Si une personne qui veut faire un testament ne doit pas estre en bon sens, de franche et libre condition, aagée de dix neuf ans accomplis, disposer de chose qui soit en sa puissance, [fol. 601v] sans sollicitation, ni induction de personne, et si le notaire ne doit pas faire mention de cet état et disposition dans ledit testament ?

4. Si un testateur n'est pas obligé de nommer specifiquement par nom et surnom tous les heritiers et legataires à peine de nullité de son testament ?

5e Si le testateur n'est pas obligé d'exhereder aussi nommément et specifiquement tous ceux, qui autrement seroient ses heritiers legitimes et ab intestat, et leur laisser au moins à titre de proprieté cinq sols faibles1 en departement de ses biens?

6. Si le testateur n'est pas obligé de faire les denominations susdites à haute voix de sa propre bouche et en presence des témoins et du notaire, ou s'il peut les donner par écrit pour ensuite estre revetues par un notaire des solemnités necessaires ?

7. Si dans tous les testamens il n'y doit pas y avoir une institution d'heritier apres les legataires, avec charge de payer et acquitter les dettes d'un defunct?

8. Si, avant la Reformation, il estoit en ce [fol. 602r] pays permis de donner son bien a son confesseur, ou s'il l'est encore dans les lieux de ce pays où la religion catolique romaine est encore professée ?

9. Si une disposition faite au profit des enfans d'un tiers, sans designer ny par leurs noms, ni par leur nombre, ni par leur sexe peut subsister par la coutume du pays?

10. Si l'on peut leguer l'usufruit d'un bien à une personne, à la charge de laisser parvenir apres sa mort la proprieté de ce bien aux enfans qu'elle a dans le tems de la disposition, et si une telle disposition ne tient pas de la substitution deffendue par la coutume du pays ?

11. Si n'estant pas fait mention dans ledit testament qu'il a esté lu et ensuite reconu par le testateur et les témoins, cela n'est pas une nullité essentielle au testament?

12. En quel degré les témoins peuvent estre parens du testateur, des legataires, de l'heritier institué et du notaire ?

13. Si un testament sollemnel doit estre [fol. 602v] revestu de la signature du testateur et des témoins avec celle du notaire ?

14. Si l'on ne doit pas faire apposer le seau de la seigneurie à l'acte que l'on veut produire sur le jour fatal, ou produire attestation qu'on la demandé à la seigneurie. 
15. Si un heritier institué et tous ceux qui ont des pretentions en la succession d'un defunt par droit de proximité ou autrement, ne doivent pas estre munis et pourvus de leurs droits, titres et informations sur le jour fatal, et s'ils ne les font pas, ils ne sont pas entierement privés et dejettés.

16. Si le jour fatal n'est pas le quarantième jour apres l'ensevelissement du defunt, et au cas que ledit defunt soit enseveli le dimanche, s'il ne faut pas demander l'investiture le samedi devant.

17. Si les absens de l'Etat de Neufchatel sont obligés de se presenter sur ledit jour fatal, et s'ils n'ont pas un an et six semaines à compter ou dès le jour de la mort du defunt, ou dès le jour qu'ils ont appris cette mort.

18. Si l'heritier institué ne doit pas presenter [fol. 603r] sur le jour fatal, or et argent sur tables pour payer les legs, ou faire offre de les payer.

19. Si un legataire est obligé sans distinction de prendre la possession en investiture sur le jours fatal en justice, tant des meubles que des immeubles à lui legués?

20. En quel tems un legataire peut il demander à l'heritier la delivrance de son leg, et dans quel tems l'heritier peut il lui opposer la prescription.

21. Si un testament nul en un point, $[\ldots] \mathrm{d}$ ne l'est pas en tous.

Mesdits sieurs du Conseil, ayant eu avis par ensemble, donnent par declaration, que de tout tems immemorial de pere à fils jusqu'a present, la coutume de Neufchastel est telle.

Assavoir, sur le 1. et 2. article que, en jugeant, on ne suit pas le droit romain, mais bien la coutume particuliere et ancienne ecrite et non ecrite usitée dans cette souveraineté.

[fol. 603v] Sur le 3me. Une personne, pour pouvoir valablement faire testament, donnation ou autre disposition de ses biens, doit avoir pour le moins 19 ans accomplis, et il doit estre de condition franche et en bon sens, sans estre induit, sollicité ni contraint, et il convient qu'elle dispose de chose qui soit en sa puissance et disposition, autrement le testament, donation ou autre disposition de bien sont deffectueux.

Sur le 4. 5. et 6. Celui, (n'ayant point d'enfans) qui veut exhereder de ses biens aucuns de ses plus proches parens, lesquels à deffaut d'enfans legitimes devroient estre selon le droit de nature ses heritiers legitimes et ab intestat s'il n'en estoit disposé autrement, il les doit specifiquement nommer, et declarer ce qu'il lègue et ordonne à un chacun d'eux en département de ses biens, l'or, argent, obligations, terres ou autres choses et pour le moins la valeur de cinq sols foibles.

Sur le 7. dans tous les testamens il y doit avoir institution d'heritier, et celui qui en consequence se porte heritier d'un defunt est obligé d'acquitter les dettes et les legs dudit defunt.

[fol. 604r] Sur le 8. Toute personne qui est de franche condition et qui n'a point d'enfans peut tester et disposer de tous ses biens et les donner à qui il lui plait, excepté à moines blancs.

Sur le 9. Une disposition faite au profit des enfans d'un tiers qui est clairement et specifiquement nommé peut subsister, sans qu'il soit necessaire d'une plus particuliere designation desdits enfans.

Sur le 10. On peut disposer d'un bien fond et donner la proprieté à une personne, et leguer l'usufruit dudit bien à une autre personne, sans que cela puisse passer pour substitution.

Sur le 11. il n'est pas de pratique que le notaire énonce que le testament a esté lu.

Sur le 12. le notaire qui reçoit un acte testamentaire, ne doit estre plus près qu'au quatrième degré de parentage avec le testateur et avec l'heritier institué. Et les témoins qui sont appellés à la passation dudit acte, ne doivent estre plus près qu'au tier en quart degré de parentage avec le notaire, avec le testateur et avec l'heritier institué par ledit testament.

[fol. 604v] Sur le 13. Il n'est pas necessaire qu'un testament passé par main de notaire en presence de cinq témoins non suspects soit signé du testateur ni des témoins.

Sur le 14. 15. 16. 17. et 18e. Celui sachant la mort d'un defunt, qui pretend avoir action à la succession d'icelui, par droit de proximité, ou en vertu d'un testament ou donation, doit demander la mise en possession et 
investiture en justice de son pretendu sur le jour des six semaines de l'ensevelissement du defunt, muni de ses droits et informations. Et quand un defunt est enseveli par un jour de dimanche, la mise en possession et investiture se doit demander le samedi precedent le jour des six semaines, qu'est la veille d'iceluy. Et en demandant la mise en possession et investiture sur ledit jour, en vertu d'un testament, donnation ou autre disposition de biens, il doit produire en ouverte justice l'acte seelé du seel de la seigneurie, ou à ce deffaut, attestation de la recherche qui en auroit esté faite. [fol. 605r] Et l'heritier testamentaire ayant produit son acte en due forme, doit sur ledit jour presenter or et argent sur table en justice pour satisfaire les legs pecuniaires en son tems, s'il y en a, ou au moins faire offre de les payer suivant l'ordonnance testamentaire. Et estant dans le lieu et ils ne s'approche pas sur ledit jour pour demander la mise en possession et investiture, estant bien certioré du trépas du defunt, il ne pourra aucunement avoir accès au bien d'icelui, ains il en est entierement et pour le tout privé et dejetté. Mais celui qui n'est pas dans le lieu, il a an et jours qu'est un an et six semaines pour s'approcher et demander la mise en possession et investiture du bien delaissé par le defunt, alors venant dans ledit tems, il peut jouir de son pretendu. Et s'il ne vient dans ledit terme d'un an et six semaines à compter du jour de l'ensevelissement, ains le laisse passer et expirer, il est entierement frustré de la succession dudit defunt et il n'en peut avoir aucune part, s'il n'en est relevé par la justice souveraine.

Sur le 19. à l'egard des legataires, la pratique n'a pas esté bien fixe jusqu'à present, les uns en ayant demandé la mise en possession et investiture et les autres ne l'ayant pas fait, sans que pour cela ils ayent perdu leurs legs.

[fol. 605v] Sur le 20. les legs portés dans un testament se doivent payer au tems et terme que le testateur l'ordonne, moyennant qu'il n'y ait aucun usufruit sur les biens d'icelui. Cependant un heritier testamentaire d'un defunt n'est pas obligé de payer les legs portés au testament au terme designé dans icelui lors qu'il y a procès ou cause intentée pour annuller ledit testament, ains seulement à la fin de la cause ou procès. Et la prescription n'arrive qu'au bout de dix ans à compter dès le jour que l'heritier est ivestu et reconu.

Sur le 21. Quand un testament est deffectueux en un point essentiel, il est censé estre deffectueux en tous.

Laquelle declaration, mesdits sieurs du Conseil ont ordonné à moy, secrétaire de ville soussigné, d'expedier en cette forme, munie du seau de la mairie et justice dudit Neufchastel, à Neufchatel le 8. may 1705.

L'original est signé par moy.

[Signature :] Jean-Jacques Favargier [Seing notarial] 